\title{
On the Galois group of Generalised Laguerre polynomials II
}

\author{
Shanta Laishram, Saranya G. Nair and T. N. Shorey
}

Dedicated to the memory of Alan Baker

Abstract. For real number $\alpha$, Generalised Laguerre Polynomials (GLP) is a family of polynomials defined by

$$
L_{n}^{(\alpha)}(x)=(-1)^{n} \sum_{j=0}^{n}\left(\begin{array}{c}
n+\alpha \\
n-j
\end{array}\right) \frac{(-x)^{j}}{j !} .
$$

These orthogonal polynomials are extensively studied in Numerical Analysis and Mathematical Physics. In 1926, Schur initiated the study of algebraic properties of these polynomials. We consider the Galois group of Generalised Laguerre Polynomials $L_{n}^{\left(\frac{1}{2}+u\right)}(x)$ when $u$ is a negative integer.

Keywords. Irreducibility, Laguerre Polynomials, Primes, Newton Polygons.

2010 Mathematics Subject Classification. Primary 11A41, 11B25, 11N05, 11N13, 11C08, $11 \mathrm{Z} 05$.

\section{Introduction}

For real number $\alpha$ and integer $n \geq 1$, the Generalised Laguerre Polynomials (GLP) is a family of polynomials defined by

$$
L_{n}^{(\alpha)}(x)=(-1)^{n} \sum_{j=0}^{n}\left(\begin{array}{c}
n+\alpha \\
n-j
\end{array}\right) \frac{(-x)^{j}}{j !} .
$$

These orthogonal polynomials have been extensively studied in various branches of analysis and mathematical physics where they play an important role. They are used in Gaussian quadrature to numerically compute integrals of the form $\int_{0}^{\infty} f(x) e^{-x} d x$. They satisfy second order linear differential equation

$$
x y^{\prime \prime}+(\alpha+1-x) y^{\prime}+n y=0, y=L_{n}^{(\alpha)}(x)
$$

and the difference equation

$$
L_{n}^{(\alpha)}(x)-L_{n}^{(\alpha-1)}(x)=L_{n-1}^{(\alpha)}(x) .
$$

Schur [Sch29], [Sch21] was the first to establish interesting and important algebraic properties of these polynomials. Schur gave a formula for the discriminant $\Delta_{n}^{(\alpha)}$ of $\mathcal{L}_{n}^{(\alpha)}(x)=n ! L_{n}^{(\alpha)}(x)$ by

$$
\Delta_{n}^{(\alpha)}=\prod_{j=1}^{n} j^{j}(\alpha+j)^{j-1}
$$

We thank episciences.org for providing open access hosting of the electronic journal Hardy-Ramanujan Journal 
and calculated their associated Galois groups. Schur [Sch29], [Sch21] showed that for every positive integer $n$, the polynomial $L_{n}^{(0)}(x)$ is irreducible and has associated Galois group the symmetric group $S_{n}$. He showed that $L_{n}^{(1)}(x)$ is irreducible for all positive integers $n$ and has associated Galois group the alternating group $A_{n}$ if $n>1$ and $n$ is odd, and $S_{n}$ otherwise. Further, he showed that the polynomials $L_{n}^{(-1-n)}(x)$ have associated Galois group $A_{n}$ if $n \equiv 0(\bmod 4)$ and $S_{n}$ otherwise. Gow [Gow89] showed that if $n$ is an even integer $>2$, then the Galois group associated with $L_{n}^{(n)}(x)$ is $A_{n}$ provided that the polynomial $L_{n}^{(n)}(x)$ is irreducible. Filaseta, Kidd and Trifonov [FKT12] proved that for every integer $n>2$ with $n \equiv 2(\bmod 4)$, the polynomial $L_{n}^{(n)}(x)$ is irreducible. For $n=2$, $L_{2}^{(2)}(x)$ is reducible but its Galois group is $A_{2}=\{e\}$. These results settled the inverse Galois problem for $A_{n}$ explicitly that for every positive integer $n>1$, there exists an explicit Laguerre polynomial of degree $n$ whose Galois group is the alternating group $A_{n}$.

From now on wards, we always assume that

$$
\alpha=u+\frac{1}{2}
$$

where $u$ is an integer. We define

$$
\mathcal{L}_{n}^{(u)}(x)=\sum_{j=0}^{n}\left(\begin{array}{l}
n \\
j
\end{array}\right)(1+2(u+n))(1+2(u+n-1)) \cdots(1+2(u+j+1)) x^{j}
$$

and observe that $(-1)^{n} \mathcal{L}_{n}^{(u)}(2 x)=2^{n} n ! L_{n}^{(\alpha)}(-x)$ and thus the irreducibility of $\mathcal{L}_{n}^{(u)}(x)$ implies irreducibility of $L_{n}^{(\alpha)}(x)$ and their associated Galois groups are same.

We recall that Hermite polynomials $H_{2 n}(x)$ and $H_{2 n+1}(x)$ are given by

$$
H_{2 n}(x)=(-1)^{n} 2^{2 n} n ! L_{n}^{\left(-\frac{1}{2}\right)}\left(x^{2}\right) \text { and } H_{2 n+1}(x)=(-1)^{n} 2^{2 n+1} n ! x L_{n}^{\left(\frac{1}{2}\right)}\left(x^{2}\right) .
$$

Schur [Sch29], [Sch21], proved that $L_{n}^{\left(-\frac{1}{2}\right)}\left(x^{2}\right)$ and $L_{n}^{\left(\frac{1}{2}\right)}\left(x^{2}\right)$ are irreducible and these imply the irreducibility of $H_{2 n}(x)$ and $H_{2 n+1}(x) / x$. We observe that $u \in\{-1,0\}$ in these results, and in [Lai15] Laishram proved that the Galois group is $S_{n}$ when $u \in\{-1,0\}$. Laishram, Nair and Shorey [LNS16],[LNS18] showed that $L_{n}^{(\alpha)}(x)$ with $\alpha=u+\frac{1}{2}$ and $1 \leq u \leq 45$ are irreducible except when $(u, n)=(10,3)$ and have associated Galois group $S_{n}$ other than in the case of $(u, n)=(10,3)$ where the Galois group is $\mathbb{Z}_{2}$. Further Nair and Shorey [NaSh18] proved that $L_{n}^{(\alpha)}(x)$ with $-18 \leq u \leq-2$ are irreducible. In [Ban18, Corollary 2.3], Banerjee showed that for each $u$ there is a finite set $S(u)$ such that $L_{n}^{(\alpha)}(x)$ has Galois group $S_{n}$ except for $n$ in $S(u)$. In this paper we explicitly determine these finite sets $S(u)$ for $u$ in the range $-18 \leq u \leq-2$ and compute the Galois group.

Theorem 1.1. Let $\alpha=u+\frac{1}{2}$ and $-18 \leq u \leq-2$. Then the Galois group of $L_{n}^{(\alpha)}(x)$ is $S_{n}$ except when $(n, u) \in\{(8,-5),(8,-6),(9,-5),(9,-6)(16,-9),(16,-10)$,

$(24,-13),(24,-14),(25,-13),(25,-14),(32,-17),(32,-18)\}$ in which cases the Galois group is $A_{n}$.

\section{Preliminaries}

We will use a result of Hajir [Haj05] which gives a criterion for an irreducible polynomial to have large Galois group using Newton polygons. We restate the result which is [Haj05, Lemma 3.1]. For an integer $x$, let $\nu(x)=\nu_{p}(x)$ be the highest power of $p$ dividing $x$ and we write $\nu(0)=\infty$.

Lemma 2.1. Let $f(x)=\sum_{j=0}^{m}\left(\begin{array}{c}m \\ j\end{array}\right) c_{j} x^{j} \in \mathbb{Q}[x]$ be an irreducible polynomial of degree $m$. Let $p$ be $a$ prime with $\frac{m}{2}<p<m-2$ such that 
(i) $\nu_{p}\left(c_{j}\right) \geq 0$ for $j=0,1, \ldots, m$,

(ii) $\nu_{p}\left(c_{0}\right)=1$,

(iii) $\nu_{p}\left(c_{j}\right) \geq 1$ for $0 \leq j \leq m-p$,

(iv) $\nu_{p}\left(c_{p}\right)=0$.

Then the Galois group of $f$ contains $A_{m}$. Further Galois group is $A_{m}$ if discriminant of $f \in \mathbb{Q}^{* 2}$ and $S_{m}$ otherwise.

Next, we state a result which we deduce from a result due to Harborth and Kemnitz and state it as a lemma so that we can use it easily.

Lemma 2.2. There exists a prime $p$ satisfying $\frac{2}{3} n \leq p<n-2$ for $n \geq 14$.

Proof. In [HaKe81], Harborth and Kemnitz proved that there exists a prime $p$ satisfying

$$
x<p \leq \frac{6}{5} x \text { for } x \geq 25 .
$$

We take $x=\frac{3}{4} n$. Then for $n \geq 34$, we conclude that there exists a prime $p$ in $\left(\frac{3}{4} n, \frac{9}{10} n\right] \subset\left[\frac{2}{3} n, n-2\right)$. For $14 \leq n \leq 33$, we check that $\left[\frac{2}{3} n, n-2\right)$ contains a prime.

Lemma 2.3. Let $r \in\{1,3\}$. The interval $(x, 1.048 x]$ contain primes congruent to $r$ modulo 4 when $x \geq 887$.

This follows from Cullinan and Hajir [CuHa12, Theorem 1] with $k=4$.

Lemma 2.4. For $-18 \leq u \leq-2$, the polynomials $L_{n}^{\left(\frac{1}{2}+u\right)}(x)$ are irreducible.

This follows from Nair and Shorey [NaSh18, Theorem 1].

\section{Galois group of $L_{n}^{(\alpha)}(x)$}

The results in this section are more general than required for the proof of Theorem 1.1. For a fixed $u$, if $n \leq 13$ we can compute Galois group directly using MAGMA. Thus we assume $n \geq 14$ and we always write

$$
v=-u
$$

Now we state our main lemma.

Lemma 3.1. Let $u \leq-2$ and $n \geq \max \{14,2 v-1\}$. Assume $\mathcal{L}_{n}^{(u)}(x)$ is irreducible. If there exists a prime $\in(2 v-3, n-2)$, then the Galois group of $\mathcal{L}_{n}^{(u)}(x)$ contains $A_{n}$.

Proof. By Lemma 2.2, there exists a prime $\in\left[\frac{2}{3} n, n-2\right)$. So we choose $p \in(2 v-3, n-2)$ such that

$$
p \geq \max \left\{\frac{2}{3} n, 2 v-1\right\} \text {. }
$$

We write

$$
c_{j}=\prod_{i=j+1}^{n}(1+2(u+i))=\prod_{i=j+1}^{n}(1+2(i-v)),
$$


$p=2 v+l$ and $l \geq-1$. We observe that $p<1+2(p-v+1)$ since $p>2 v-3$. Further

$$
3 p>2(n-v)+3
$$

since $p \geq \frac{2}{3} n$ and $v \geq 2$. Therefore, we conclude that $p \nmid c_{p}$.

Next we show that $\nu_{p}\left(c_{j}\right)=1$ for $0 \leq j \leq n-p$. For $0 \leq j \leq n-p$, the smallest factor in $\prod_{i=j+1}^{n}(1+2(i-v))$ occurs with $j=0$ and $i=1$ and this is $3-2 v$. The largest occurs with $i=n$ and this is $2(n-v)+1$. Since $-p<3-2 v$ and $2(n-v)+1<3 p$, this product can only involve one factor of $p$. Furthermore, for $0 \leq j \leq n-p$, this product is a product of at least $p$ consecutive odd numbers, so $p$ divides it. So $p$ exactly divides $c_{j}$ for $j$ in this range. Therefore we conclude that the Galois group of $\mathcal{L}_{n}^{(u)}(x)$ contains $A_{n}$ by Lemma 2.1 .

We recall that the discriminant $\Delta_{n}^{(u)}$ of $\mathcal{L}_{n}^{(u)}(x)$ is given by

$$
\Delta_{n}^{(u)}:=\prod_{j=2}^{n} j^{j}\left(\frac{2 u+1+2 j}{2}\right)^{j-1}
$$

Lemma 3.2. Let $2 n \geq \max \{64,2 v-5\}$. Then, $\Delta_{n}^{(u)}$ is not a square for all pairs of $(n, u)$ satisfying above conditions.

Proof. Given a positive integer $n$, let $n_{o}$ and $n_{e}$ denote the largest odd and even number less than or equal $n$ respectively. Then

$$
\Delta_{n}^{(u)}=1 \cdot 3 \cdot 5 \cdots n_{o} \cdot(2 u+1+4)(2 u+1+8) \cdots\left(2 u+1+2 n_{e}\right) \times 2^{\frac{-n_{e}}{2}} \times
$$

where $\square$ denotes a term in $\mathbb{Q}^{* 2}$. If $n \equiv 2,3(\bmod 4)$, then $\frac{n_{e}}{2}$ is odd and hence $\Delta_{n}^{(u)}$ is not a square. Hence $n \equiv 0,1(\bmod 4)$. We consider

$$
D_{v}=1 \cdot 3 \cdot 5 \cdots n_{o}(-2 v+1+4)(-2 v+1+8) \cdots\left(-2 v+1+2 n_{e}\right) .
$$

It suffices to show that $D_{v}$ is not a square. Let $r \in\{1,3\}$ be such that $-2 v+1 \equiv r(\bmod 4)$. Then

$$
D_{v}=1 \cdot 3 \cdots n_{o}(-2 v+1+4)(-2 v+1+8) \cdots(r-4) \cdot r \cdot(r+4) \cdots\left(-2 v+1+2 n_{e}\right) .
$$

Let $p_{0}$ be the largest prime with $\frac{2}{3} n \leq p<n$ and $p \equiv 3 r(\bmod 4)$. We take $x=\frac{2}{3} n$ and $n>1330$ in Lemma 2.3 to conclude that the interval $\left[\frac{2}{3} n, n-2\right)$ contain both primes congruent to 1 and 3 modulo 4. We check that for $32 \leq n \leq 1330$, the interval $\left[\frac{2}{3} n, n-2\right)$ contain both primes congruent to 1 and 3 modulo 4. Then $p_{0} \| 1 \cdot 3 \cdot 5 \cdots n_{o}$. Further $3 p_{0}>2 n>2 n_{e}-2 v+1$ and $2 v-5 \leq 2 n<3 p_{0}$. Since $p \equiv 3 r(\bmod 4)$ and $-2 v+1 \equiv r(\bmod 4)$, the above conditions imply that $p_{0} \| D_{v}$ and hence $D_{v}$ is not a square. Therefore $\Delta_{n}^{(u)}$ is not a square.

The following result is obtained by direct computation using MAGMA.

Lemma 3.3. Let $2 \leq n \leq 40$ and $-18 \leq u \leq-2$. Then the Galois group of $\mathcal{L}_{n}^{(\alpha)}(x)$ is $S_{n}$ except when $(n, u) \in\{(8,-5),(8,-6),(9,-5),(9,-6)(16,-9),(16,-10),(24,-13),(24,-14),(25,-13),(25,-14)$, $(32,-17),(32,-18)\}$ in which cases the Galois group is $A_{n}$.

We now prove Theorem 1.1. 


\section{Proof of Theorem 1.1:}

Let $-18 \leq u \leq-2$. Then $\mathcal{L}_{n}^{(u)}(x)$ are irreducible by Lemma 2.4. Further, by Lemma 3.3, we can always assume that $n \geq 41$. Since $v \leq 18$, we have $n \geq 2 v-1$. If $n \geq 50$, then $2 v-3<\frac{2}{3} n$ and hence using Lemma 2.2, we conclude that there exists a prime in $(2 v-3, n-2)$. If $41 \leq n \leq 49$, then the prime 37 is in this interval as $2 v-3 \leq 33$. Hence, by Lemmas 3.1 and 3.2, the Galois group of $\mathcal{L}_{n}^{(u)}(x)$ is $S_{n}$.

\section{Acknowledgements}

We would like the thank the referee for useful comments in an earlier version of the paper. The first named author(SL) acknowledges the support of the MATRICS Grant of SERB, DST, India.

\section{References}

[Ban18] P. Banerjee, On Galois groups of a one-parameter orthogonal family of polynomials, Acta Arith., 183 (2018), 1-34.

[CuHa12] J. Cullinan and F. Hajir, Primes of prescribed congruence class in short intervals, Integers, 12 (2012), Paper No. A56, 4 pp.

[FKT12] M. Filaseta, T. Kidd and O. Trifonov, Laguerre polynomials with Galois group $A_{m}$ for each $m$, J. Number Theory, 132 (2012), 776-805.

[Gow89] R. Gow, Some generalized Laguerre polynomials whose Galois groups are the Alternating groups, J. Number Theory, 31 (1989), 201-207.

[Haj05] F. Hajir, On the Galois group of generalized Laguerre polynomials, J. Théor. Nombres Bordeaux, 17 (2005), 517-525.

[HaKe81] H. Harborth and A. Kemnitz, Calculations for Bertrand's postulate, Math. Mag., 54 (1981), 33-34.

[Lai15] S. Laishram, On the Galois groups of generalized Laguerre Polynomials, Hardy Ramanujan Journal, 37 (2015), 8-12.

[LNS16] S. Laishram, S. G. Nair and T. N. Shorey, Irreducibility of Generalized Laguerre Polynomials $L_{n}^{\left(\frac{1}{2}+u\right)}(x)$ with integer u, J. of Number Theory, 160 (2016), 76-107.

[LNS18] S. Laishram, S. G. Nair and T. N. Shorey, Irreducibility of extensions of Laguerre Polynomials, Funct. Approx. Comment. Math. (to appear).

[NaSh18] S. G. Nair and T. N. Shorey, Irreducibility of Generalised Laguerre Polynomials $L_{n}^{\left(\frac{1}{2}+u\right)}\left(x^{2}\right)$ with $-18 \leq u \leq-2, A c t a$ Arithmetica, 184 (2018), 363-383.

[Sch29] I. Schur, Einige Sätze über Primzahlen mit Anwendungen auf Irreduzibilitätsfragen, II, Sitzungsber. Preuss. Akad. Wiss. Berlin Phys.-Math. Kl., 14 (1929), 370-391.

[Sch21] I. Schur, Affektlose Gleichungen in der Theorie der Laguerreschen und Hermitschen Polynome, J. Reine Angew. Math., 165 (1931), 52-58.

\section{Shanta Laishram}

Stat-Math Unit, Indian Statistical Institute

7, S. J. S. Sansanwal Marg

New Delhi, 110016, India

e-mail: shanta@isid.ac.in

\section{Saranya G. Nair}

Department of Science and Mathematics

Indian Institute of Information Technology Guwahati

Bongora, Assam 781015, India

e-mail: saranya@iiitg.ac.in

\section{T. N. Shorey}

National Institute of Advanced Studies, IISc Campus

Bangalore, 560012, India

e-mail:shorey@math.iitb.ac.in 